\title{
Liver and Intestine Transplantation in the United States, 1996-2005
}

\author{
E. A. Pomfret ${ }^{a}$ * , J. P. Fryer ${ }^{b}$, C. S. Sima ${ }^{c}$, J. R. \\ Lake $^{d}$ and R. M. Merion ${ }^{\mathrm{e}}$ \\ a Lahey Clinic Medical Center, Burlington, Massachusetts, \\ USA \\ ${ }^{\mathrm{b}}$ Northwestern University Feinberg School of Medicine, \\ Chicago, Illinois, USA \\ 'Scientific Registry of Transplant Recipients, Arbor \\ Research Collaborative for Health, Ann Arbor, Michigan, \\ USA \\ 'University of Minnesota Medical Center Fairview, \\ Minneapolis, Minnesota, USA \\ e Scientific Registry of Transplant Recipients, University of \\ Michigan, Ann Arbor, Michigan, USA \\ * Corresponding author: Elizabeth A. Pomfret, \\ Elizabeth.A.Pomfret@lahey.org
}

The number of liver transplants performed yearly has slowly and steadily increased over the last 10 years, reaching 6441 procedures in 2005 . The number of living donor liver transplants performed rose steadily from 1996 to 2001, when it peaked at 519; since 2003 there have been approximately 320 such procedures performed each year. The continual increase in the size of the waiting list for a liver transplant, which peaked in 2001 at 14897 patients, was interrupted in 2002 by the implementation of the allocation system based on the model for end-stage liver disease and pediatric end-stage liver disease (MELD/PELD). Activity in all areas of intestinal transplantation continues to increase. One-year patient and graft survival following intestinealone transplantation now seem to be superior to outcomes following liver-intestine transplantation. Other topics covered here include the recent 'Share 15' component of the MELD allocation system; liver transplantation following donation after cardiac death; simultaneous liver-kidney transplantation and waiting list and post-transplant outcomes for both liver and intestine transplantation, broken out by a variety of clinical and demographic factors.

Key words: Allocation policy, MELD/PELD, OPTN, SRTR, survival rates, waiting list

\section{Introduction}

The shortage of organs for transplantation continues to be a major impediment to providing optimal treatment for patients with end-stage organ failure. It is particularly acute in patients requiring extra-renal organs for which dialysisequivalent therapies are nonexistent and the prospect of death while waiting for a transplantable organ is a realistic possibility.

\section{Liver Waiting List}

The continual increase in the size of the waiting list for a liver transplant, which peaked in 2001 at 14897 patients, was interrupted in 2002 by the implementation of the allocation system based on the model for end-stage liver disease and pediatric end-stage liver disease (MELD/PELD). The important drop registered between 2001 and 2003 in the number of candidates actively waiting for a liver transplant $(15 \%)$ was followed by a slow increase over the last 2 years (12822 candidates in 2005, compared to 12650 in 2003); the exact significance of this upward trend is unclear (Figure 1). Conversely, the percentage of listed patients on the inactive waiting list has remained relatively constant at approximately $25 \%$, with the majority of patients (78\%) on the inactive list in 2005 being listed for two or more years.

\section{Demographic factors}

Age: The age distribution among patients active on the waiting list underwent significant changes over the last 10 years. While in 1996 most candidates were equally distributed between the 18-49 and 50+ age categories, the group aged 50-64 alone now makes up nearly $60 \%$ of the patients active on the waiting list (Figure 2). This shift most likely reflects the changing demographics of U.S. society, which has an increasingly older population. In contrast, the number of patients 18-49 years old remained approximately the same over the decade, but this age range's percentage of all active waiting list patients declined from $47 \%$ in 1996 to $27 \%$ in 2005 . A similar trend is seen among pediatric $(<18)$ candidates, who continue to represent less than $5 \%$ of the waiting list, while older adults $(65+)$ reached $11 \%$ of the waiting list for the first time in 2005

Race and ethnicity: White candidates continue to make up most of the liver waiting list, but their percentage has slowly declined (from $77 \%$ in 1996 to $72 \%$ in 2005), currently reaching a level close to the one observed in the general population. The death rate for whites on the waiting list is 123 deaths/1000 patient-years (PY) at risk, very close to the death rate for the overall waiting list. The numbers of African American and Asian patients on the waiting 


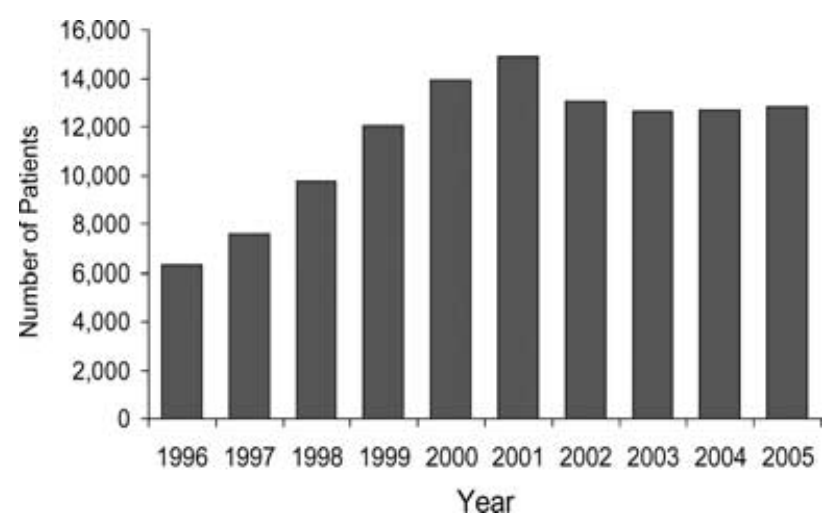

Source: 2006 OPTN/SRTR Annual Report, Table 9.1a.

Figure 1: Number of patients on the liver waiting list, active at year-end, 1996-2005.

list have remained relatively constant (approximately $7 \%$ and $5 \%$ of the waiting list, respectively). While other races have increased their number of active candidates by 90 $131 \%$, the number of Hispanics active on the waiting list has nearly tripled in the last 10 years. Asian candidates continue to have the lowest mortality rate among all races $(87$ deaths/1000 PY at risk), while the African Americans have the highest rate (154 deaths/1000 PY at risk).

Gender: The number of females active on the waiting list continues to be lower than the number of males, and even higher numbers of male registrations have widened this gap further. In 2004 and 2005, women made up approximately $40 \%$ of the active list, down from $44 \%$ in 1996. The death rate on the waiting list continues to be lower for females (119 deaths/1000 PY at risk) than for males (130 deaths/1000 PY at risk).

\section{Medical factors}

Diagnosis: The distribution of diagnoses at listing has been very stable since 2000. Noncholestatic liver disease remains the largest single diagnostic category, represent-

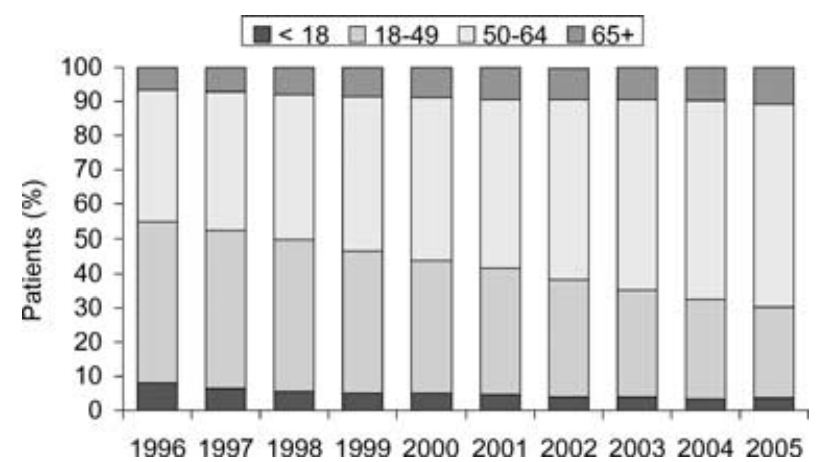

Source: 2006 OPTN/SRTR Annual Report, Table 9.1a.

Figure 2: Age distribution of patients on the liver waiting list, active at year-end, 1996-2005. ing about $72 \%$ of the waiting list. Although the percentage of patients with noncholestatic liver disease has increased slightly (72\% in 2005 vs. 67\% in 1996) the absolute number of patients with this diagnosis more than doubled over the decade. Biliary atresia has consistently been associated with the lowest mortality risk (52 deaths/1000 PY in 2005), while patients diagnosed with acute hepatic necrosis (165 deaths/1000 PY at risk), malignant neoplasm (132 deaths/1000 PY at risk) or metabolic diseases (123 deaths/1000 PY at risk) remain the diagnoses with the highest death rates.

Previous transplant: The proportion of candidates awaiting liver transplantation who underwent a previous transplant of any kind steadily decreased between 1996 and 2004 (Figure 3). However, in 2005 the percentage of retransplant candidates on the active liver transplant waiting list increased for the first time in the decade-even though the increase was minor $(3.3 \%$ in 2005 , compared to $3.1 \%$ in 2004). The percentage of patients listed for a second liver transplant decreased from 5\% in 1996 to $3 \%$ in 2005, primarily because the total number of wait-listed patients rose over the same period (6280 at year-end 1996 vs. 12822 at year-end 2005).

\section{Waiting list death rates}

Very young candidates ( $<1$ year) have the highest mortality on the waiting list (722 deaths/1000 PY at risk), followed by pediatric candidates aged $1-5$ years (186 deaths/1000 $\mathrm{PY}$ at risk). Among candidates six and older at listing, the death rates increased with age, with the 6- to 10-year-olds having the lowest mortality rate (39 deaths/1000 PY at risk) and the group older than 65 years having the highest (158 deaths/1000 PY at risk).

\section{Liver allocation system}

The current allocation system gives priority to candidates listed as Status $1 \mathrm{~A}$ or $1 \mathrm{~B}$, followed by non-Status 1

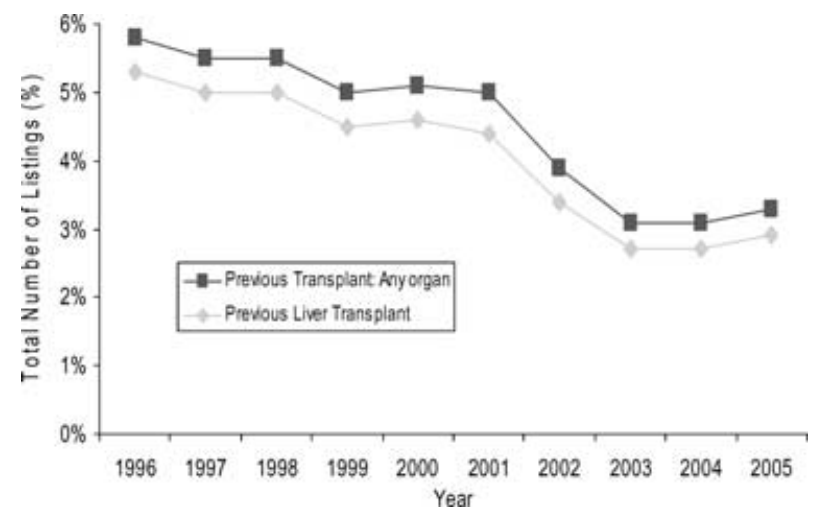

Source: 2006 OPTN/SRTR Annual Report, Table 9.1a.

Figure 3: Percentage of candidates on the liver waiting list with a previous transplant by year, 1996-2005. 
candidates in decreasing order of MELD/PELD score. Additionally, exceptions to the usual listing order are granted for patients with hepatocellular carcinoma (HCC). Initially, T1 (one tumor $\leq 1.9 \mathrm{~cm}$ ) and T2 (one nodule $2.0-5.0 \mathrm{~cm}$; two or three nodules all $<3.0 \mathrm{~cm}$ ) tumors were eligible for exception. Concerns that T1 lesions were difficult to diagnose accurately-and that HCC patients were perhaps being given excessive priority-led to a policy change in 2004 allowing exception for only those patients with T2 tumors.

Since August 2005, following a change in policy for Status 1 listing, adult and pediatric candidates must have fulminant hepatic failure, primary nonfunction of a transplanted liver, hepatic artery thrombosis or acute decompensated Wilson's disease to be listed as Status 1A. Status 1B is exclusively for pediatric patients with acute decompensation of chronic liver disease. The total number of candidates listed as Status 1 ( $A$ or B) did not change appreciably in 2005 compared to previous years (17 patients at year-end), representing only $0.1 \%$ of the total number of active patients on the waiting list in a snapshot at year-end. The apparently low percentage is explained by the fast rate of events among such candidates: $54 \%$ of the patients listed with Status 1 in 2004 and 2005 were transplanted within 15 days of listing, an additional 12\% recovered and 16\% died or were considered too sick to be transplanted. Only $9 \%$ of patients were still waiting for a transplant 15 days after being designated Status 1. Most of the transplants, recoveries and deaths occurred during the first 7 days after listing. The risk of death on the waiting list while a Status 1 candidate was 6619 deaths/1000 PY at risk, more than 50 times higher than the average risk for the waiting list overall.

The rest of the waiting list consisted of candidates listed at their calculated MELD/PELD score. Based on end-ofyear snapshots of the waiting list, the distribution of MELD scores among adult candidates has been remarkably stable since the MELD system was implemented (Figure 4). Between $42 \%$ and $46 \%$ of the candidates had a MELD score less than 11 , while $47-51 \%$ of the candidates had a MELD score between 11 and 20. At the end of each year from 2002 to 2005, 4\% of adult candidates were listed at a MELD score greater than 20. A different trend can be observed among pediatric candidates, where, before 2005, the overwhelming majority (80-82\%) had a PELD score of 10 or less, while only $14-17 \%$ had a PELD score of $11-$ 20. In 2005 the distribution shifted toward higher PELD scores $166 \%$ of the pediatric candidates listed at PELD $<11,26 \%$ at PELD $11-20,8 \%$ listed at PELD $>20$ ). As expected, the unadjusted risk of death was higher for those with higher MELD/PELD scores. For adults, there were 34 deaths/1000 PY at MELD 6-10, 97 deaths/1000 PY at MELD 11-20, 643 deaths/1000 PY at MELD 21-30, and 4220 deaths/1000 PY at MELD > 30. Candidates for a liver transplant with a hepatocellular carcinoma T1 (HCC T1) exception faced an unadjusted risk of dying of 90/1000 PY at

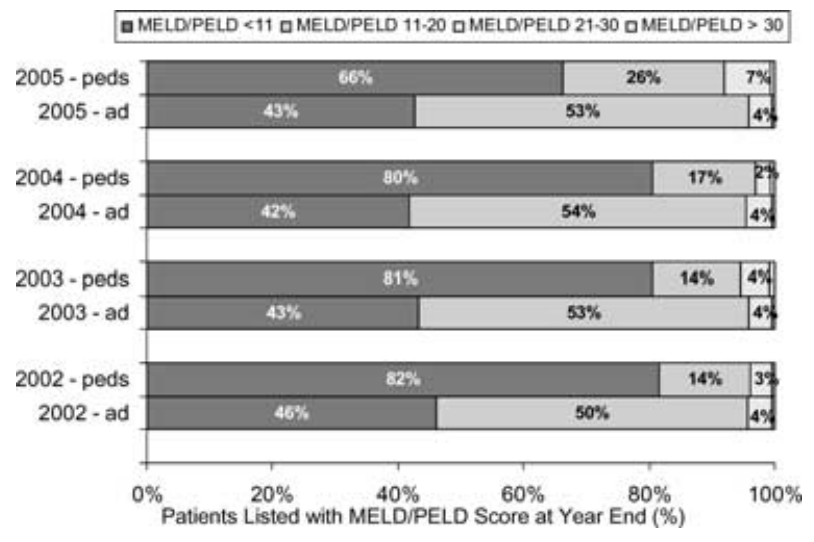

*Source: 2006 OPTN/SRTR Annual Report, Table 9.1a.

Figure 4: Distribution of MELD/PELD status among patients on the liver waiting list, at year-end, 2002-2005.

risk, while those candidates with an HCC T2 exception had a risk of 149 deaths/1000 PY at risk.

A recent analysis of Scientific Registry of Transplant Recipients (SRTR) data examined geographic differences in MELD score, risk of death on the waiting list and transplant rates (1). Roberts et al. found that the average MELD and risk of death varied somewhat by region, but that transplant rates varied much more by region, with 7 of the nation's 11 regions having transplant rates significantly different from the national average. For more discussion of regional differences in MELD score, see the section on 'Share 15', below, and the paper by Roberts et al.

\section{Deceased Donor Liver Recipients}

The total number of transplants performed yearly has increased slowly and fairly steadily over the last 10 years, reaching 6441 procedures in 2005 . This rise is mainly attributable to deceased donor liver transplantation (DDLT), the number of which increased by 30\% since 2001 after rising more slowly for the previous decade.

\section{Demographic factors}

Distribution of demographic factors among deceased donor transplant recipients follows the waiting list distribution closely.

Age: The number of pediatric recipients of DDLT increased modestly from 472 in 1996 to 509 in 2005, but the percentage of deceased donor pediatric transplants decreased from $12 \%$ to $8 \%$ over the same period. This shift mirrors the trend in waiting list registrations. The number of adult patients aged $18-49$ receiving a DDLT remained relatively stable over the decade, but the percentage of total DDLT decreased considerably, dropping from $44 \%$ in 
1996 to $29 \%$ in 2005 . In contrast, the number of adults 50 and older who were transplanted more than doubled over the same period; this group now accounts for $62 \%$ of all DDLT performed. In 1996, 276 over 65 recipients were transplanted; by 2005, the number had increased to 604 .

Gender, race, ethnicity, blood type: The number of males receiving a DDLT steadily increased over the past decade, rising from $58 \%$ in 1996 to $67 \%$ in 2005 . This change is likely due to the increased prevalence of hepatitis $C$ as the etiology of end-stage liver disease and the preponderance of males with this diagnosis. The percentage of whites who received DDLT decreased from $76 \%$ in 1996 to $72 \%$ in 2005. Over the last 10 years, African Americans represented a steady $9-10 \%$ of all the recipients of a DDLT. The distribution of blood groups among recipients of DDLT has remained constant and reflects the distribution of blood types in the general population. Of deceased donor recipients transplanted in 2005, approximately $45 \%$ were type O, 38\% were type A, 12\% were type B and $5 \%$ were type $A B$.

Insurance: More than half of the recipients of a liver transplant have private insurance as their main source of payment. A decade ago, there were more than twice as many recipients with a private insurance than recipients with public insurance, for both deceased donor and living donor liver transplants (LDLT). In 2005, the percentage of DDLT recipients with private insurance as their main source of payment decreased to $58 \%$, while the percentage of LDLT increased to $74 \%$.

\section{Medical factors}

This year's report does not examine trends in immunosuppressive therapy for liver transplantation. For an extensive examination of this topic, see Meier-Kriesche et al. in the 2005 SRTR Report on the State of Transplantation (2).

Previous transplant: There has been a slight decline in the percent of DDLT recipients who had a prior transplant of any kind. In 1996, 12\% had received a previous transplant; in 2005, this percentage decreased to $10 \%$. For an extended discussion of liver retransplantation, see 'Repeat Organ Transplantation in the United States, 1996-2005', an accompanying article in this report (3).

Partial liver grafts: The number of transplants using a partial or split liver increased by almost $40 \%$ over the last 10 years (228 in 2005, compared to 165 in 1996) (Figure 5). However, partial and split-liver transplants now represent less than $4 \%$ of the total number of liver transplants.

Diagnosis: DDLT patients with noncholestatic cirrhosis as the primary indication for transplant represented approximately $62 \%$ of DDLT recipients, a percentage similar to that seen for the last decade. The rest of the recipients were

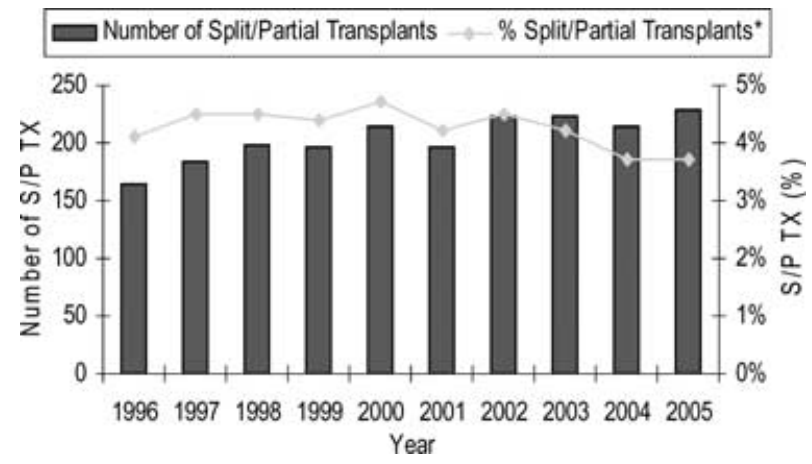

As percentage of the total number of liver transplants Source: 2006 OPTN/SRTR Annual Report, Table 9.4a.

Figure 5: Number and percentage of split/partial transplant recipients by year, 1996-2005.

listed with a diagnosis of cholestatic liver disease (7\%, continually decreasing over the last 10 years), malignant neoplasm (8\%, which experienced a significant increase in 2002, following the implementation of the exception score system that gives additional MELD points to candidates listed with $\mathrm{HCC})$, acute hepatic necrosis $(8 \%)$, biliary atresia (3\%), metabolic diseases (3\%) or other diagnosis (8\%). The number of patients transplanted for HCC has shown a steady increase since the implementation of the MELD system that grants exception points for this indication. In $2001,3 \%$ of DDLT were for HCC. In 2003, following the implementation of the MELD system, this number rose to $6 \%$; it has risen each subsequent year. The proportion of patients transplanted as Status 1 has slowly decreased since the implementation of the MELD system in 2002. In 2005, $9 \%$ of DDLT recipients and $7 \%$ of LDLT recipients were Status 1 at the time of transplant.

\section{Posttransplant death rates}

For DDLT, recipient death rates during the first year following transplantation declined, reaching the lowest value in the last decade in 2004 (Figure 6). In 2004, older adults

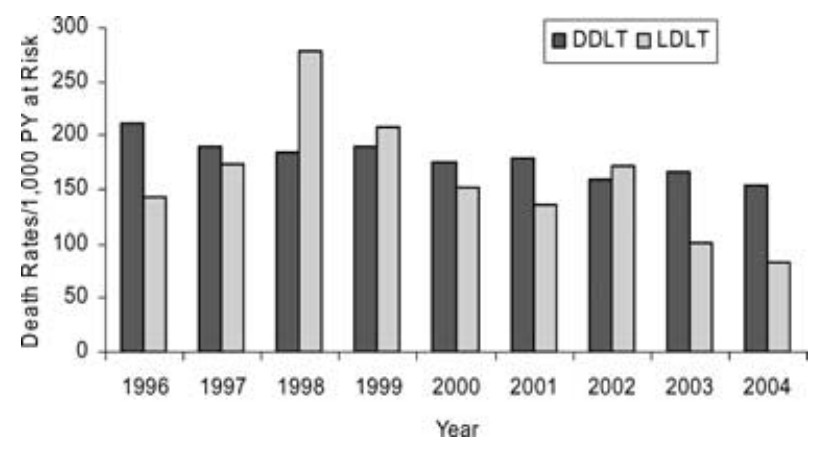

Source: 2006 OPTN/SRTR Annual Report, Table 9.7a.

Figure 6: Death rates at 1 year following transplantation by year, 1996-2004. 


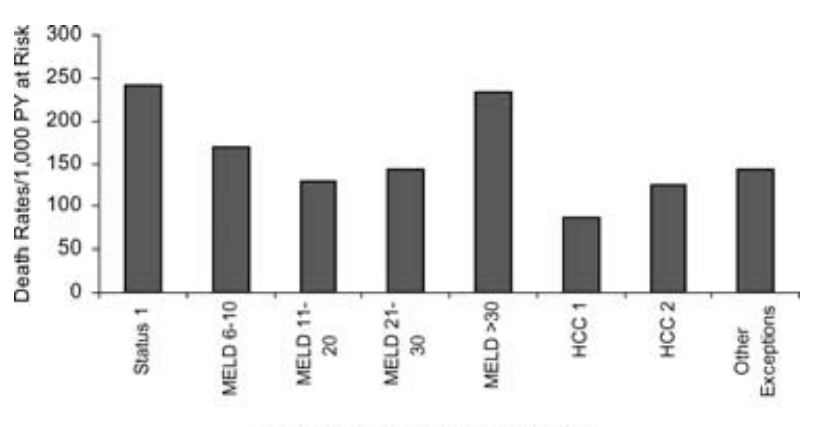

Waiting List Status at Transplant

Source: 2006 OPTN/SRTR Annual Report, Table 9.7a.

Figure 7: Death rates at 1 year following transplantation by severity of disease, 2004.

(65+ years) experienced the highest death rates (226 deaths/1000 PY at risk), while adolescents (11-17 years) had the lowest death rates (60 deaths/1000 PY at risk) among all recipients of a DDLT. Asians continue to have the lowest posttransplant death rates among all racial groups (in 2004, 136 deaths/1000 PY at risk). Males experienced a higher mortality rate than females in the first year following DDLT (149 vs. 160 deaths per 1000 PY).

Posttransplant death rates among recipients transplanted at Status 1 dropped continuously over the last 10 years. Adults transplanted at low MELD continued to experience higher 1-year death rates than those transplanted at intermediate MELD scores (Figure 7).

\section{Evaluation of an Allocation Policy Change to Share Livers Regionally to Candidates with Meld Scores Greater Than 15}

Since 2002, deceased donor liver allocation in the United States has been based on the risk of candidate death in the absence of a transplant (medical urgency) using MELD. Due to limitations of the size of the initial sharing area (local donation service area, or DSA), many livers were trans-

Table 1: Comparison of allocation rules for deceased donor livers

\begin{tabular}{ll}
\hline February 2002 to January 2005 & \multicolumn{1}{c}{ January 2005 to present } \\
\hline Local-Status 1 & Local-Status 1 \\
Regional-Status 1 & Regional-Status 1 \\
Local-MELD/PELD & Local-MELD/PELD $\geq \mathbf{1 5}$ \\
Regional-MELD/PELD & Regional-MELD/PELD $\geq \mathbf{1 5}$ \\
& Local-MELD/PELD $<\mathbf{1 5}$ \\
& Regional-MELD/PELD $<\mathbf{1 5}$ \\
National-Status 1 & National-Status 1 \\
National-MELD/PELD & National-MELD/PELD
\end{tabular}

Source: OPTN. Boldface indicates the updated Share 15 system, which went into effect January 12, 2005; the other rules were unchanged. planted locally into recipients with low MELD scores when candidates with higher MELD scores were waiting in adjoining DSAs within the same region. Previous analyses have shown that recipients transplanted at MELD $<15$ do not have a significant survival benefit from transplantation (4). In response to these observations, a change in national liver allocation policy was approved and became effective in January 2005 (5). The allocation primacy of local and regional Status 1 candidates was unchanged. Under the new policy, after Status 1 candidates, offers of livers are to be made to candidates with MELD $\geq 15$ outside the procuring DSA (but within the same region) if there are no local candidates with MELD scores $\geq 15$. Donor livers are allocated to local candidates with MELD $<15$ only if there are no regional candidates with $M E L D \geq 15$ (Table 1).

To examine the early effects of this policy change, the SRTR analyzed liver transplant data from before and after the sharing policy ('Share 15'). The analysis was presented in a preliminary form at the International Liver Transplantation Society meeting in Milan in 2006 (6). The pre-Share 15 era included 5301 DDLT from January 12, 2004, to January 11, 2005; the post-Share 15 era included 5541 transplants from January 12, 2005, to January 11, 2006.

The proportions of candidates with MELD $\geq 15$ at the time of listing in the two periods were very similar $(75.8 \%$ vs. $76.3 \%$, respectively). The distribution of reasons for removal from the liver waiting list showed a modest shift toward a higher proportion removed for deceased donor transplant and a lower proportion removed for death (Figure 8).

There was a $36 \%$ drop in the proportion of liver transplant recipients with MELD score $<15$ ( $n=627 ; 11.8 \%$ vs. $n=423 ; 7.6 \%$ for pre-Share 15 vs. post-Share 15 , respectively). The proportion of transplants to recipients with MELD $\geq 15$ increased in every region and in most DSAs (Figures 9 and 10). DSAs with lower percentages of recipients with MELD $\geq 15$ before the policy change had the

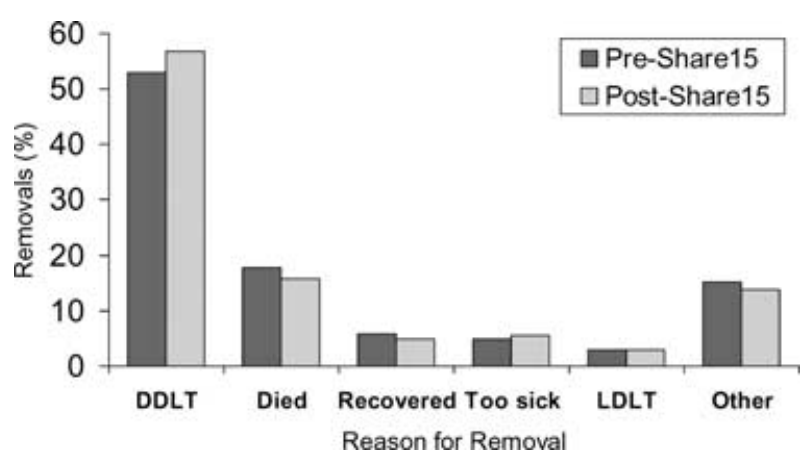

Source: SRTR Analysis, May 2006.

Figure 8: Distribution of reasons for removal from the liver waiting list. 


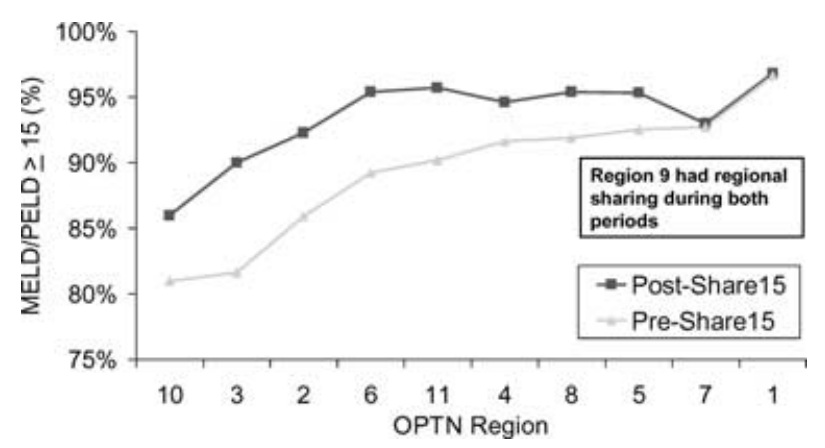

Source: SRTR Analysis, May 2006.

Figure 9: Percentage of transplant recipients with MELD/PELD ${ }^{3} 15$ by OPTN region.

largest increases after the policy was changed, reducing variability in MELD at transplant across the country. The number of DSAs where at least $90 \%$ of the liver transplants were in recipients with MELD $\geq 15$ increased from 27 to 42 (55-86\%; $p=0.017$ ) (Figure 11).

Most interestingly, despite major changes in the MELD scores of recipients and marked reductions in the number of low-MELD transplants being performed after the implementation of the new policy, there was almost no change in the number of livers shared outside the local DSA under the new system. Specifically, there was no change in the proportion of locally transplanted or regionally transplanted livers. This suggests that the policy goals were realized through behavioral changes at the local level. Decisions at the local DSA level to accept donor livers for high-MELD candidates that would have previously been reserved for lower-MELD candidates (by turning down such offers for the higher-MELD candidates) may explain this phenomenon, though this has not been examined. Methodologies to examine organ and offer acceptance are now being developed that will allow for further dissection of this process. In the meantime, the policy appears to have had its intended effect.

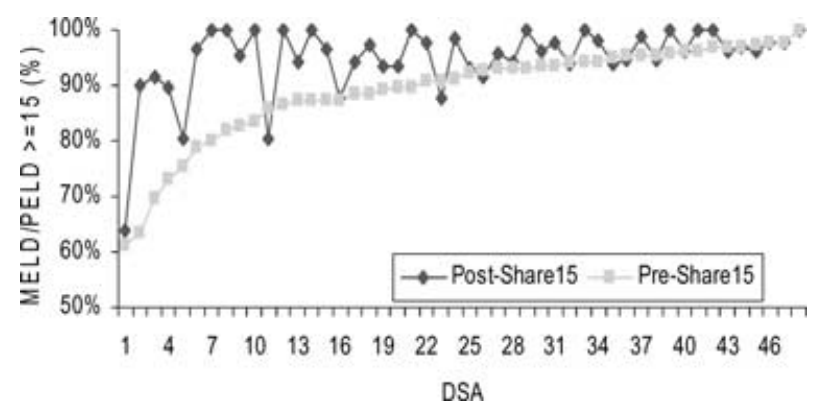

Source: SRTR Analysis, May 2006.

Figure 10: Percentage of transplant recipients with MELD/PELD $\geq 15$, by DSAs performing liver transplants.

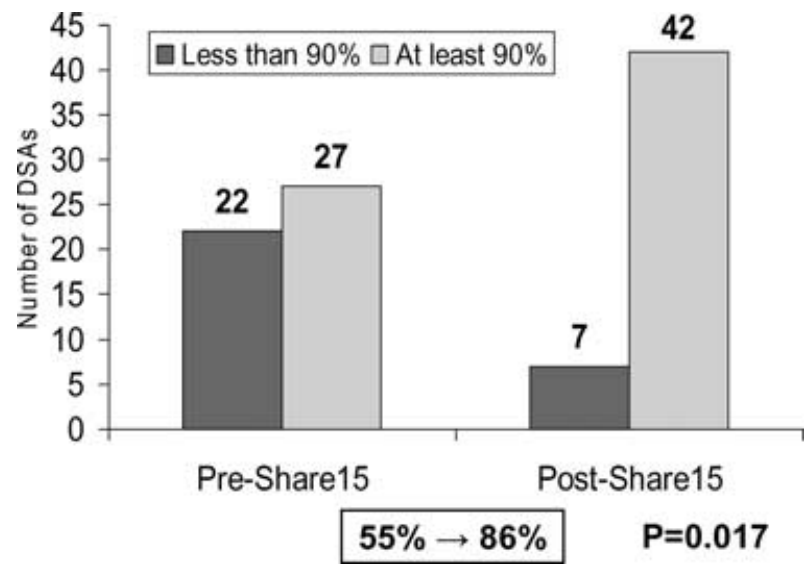

Source: SRTR Analysis, May 2006.

Figure 11: Number of DSAs with at least $90 \%$ of transplants at MELD/PELD $\geq 15$.

\section{Living Donor Liver Transplantation}

The overall incidence of LDLT increased each year between 1996 and 2001, peaking at $10 \%$ of the total number of liver transplants performed (Figure 12). Since then, the percentages have decreased to $5 \%$ of the total. Nationally, this decline may reflect the well-publicized deaths of two U.S. donors as well as the introduction of the MELD/PELD system and the increased use of so-called expanded criteria liver donors. However, the incidence of LDLT varies greatly throughout the country. In regions where the average MELD score for DDLT is 25 or greater (Regions 1, 5, 7 and 9), the percentage of patients undergoing LDLT continues to increase and currently represents approximately $10 \%$ of all liver transplants. Between 2001 and 2005, the percentage of LDLTs in regions 1, 5, 7 and 9 ranged from $9 \%$ to $13 \%$. These above-average percentages may reflect relatively low regional numbers of deceased donors and a consequent difficulty in obtaining deceased donor

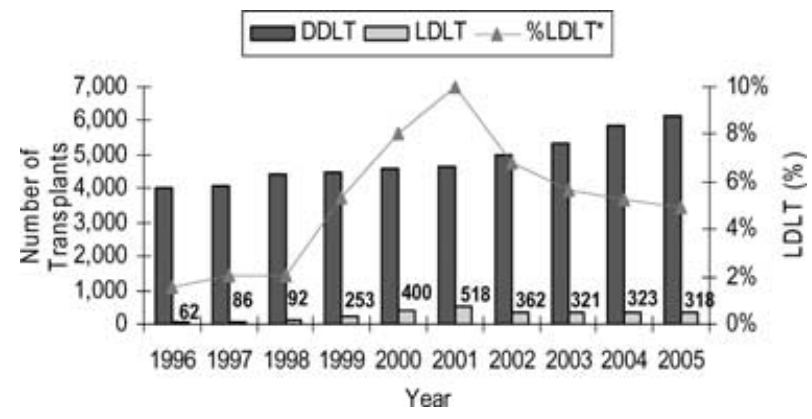

*LDLT as percentage of the total number of liver transplants Source: 2006 OPTN/SRTR Annual Report, Tables 9.4a and 9.4b.

Figure 12: Number of liver recipients by type of transplant and year, 1996-2005. 


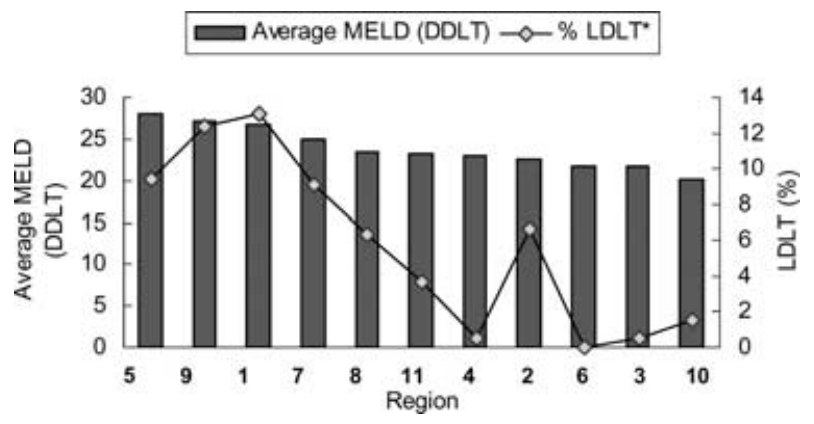

*LDLT as percentage of the total number of liver transplants Source: SRTR Analysis, May 2006.

Figure 13: Average MELD at deceased donor liver transplant and percentage of LDLT, by OPTN region.

livers for patients in a timely manner. For example, Region 1 had a total of 584 deceased donors for this period and an average MELD at time of transplant of 26.7. The only other region to have less than 1000 deceased donors in this period was Region 6-which performed a total of 637 transplants, all from deceased donors, but with an average MELD of 21.7 (Figure 13). Another possible explanation for the regional variance in LDLT is regional difference in expertise and preference.

The majority of living liver donors are genetically related to their recipients. However, the nature of the relationship has changed over time (Figure 14). In 1996, $87 \%$ of living donors were parents donating to their children; in 2005 , this percentage had decreased to $16 \%$. Conversely, in 2005, child-to-parent (27\%) and sibling-to-sibling (15\%) donations made up the largest groups of living donors, followed by other related donors (10\%). The percentage of spousal and other genetically unrelated donors has also increased, rising from 3\% in 1996 to $18 \%$ in 2005.

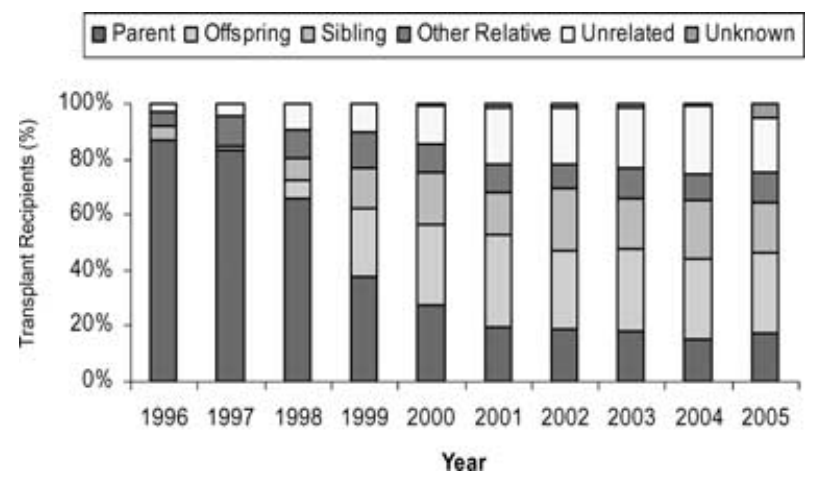

Source: 2006 OPTN/SRTR Annual Report, Table 9.4b.

Figure 14: Relation of donor to recipient for LDLT by year, 1996-2005.

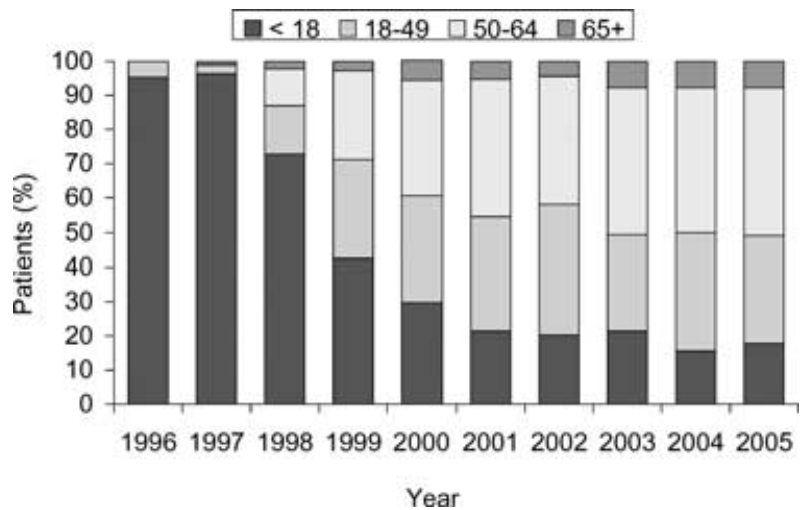

Source: 2006 OPTN/SRTR Annual Report, Table 9.1a.

Figure 15: Distribution of age among living donor transplants by year, 1996-2005.

\section{Living donor recipients}

The number of LDLT performed annually rose steadily from 1996 to 2001, when it peaked at 519. The number of LDLT dropped sharply afterward; since 2003 there have been approximately 320 such procedures performed each year. Two reasons commonly cited for this drop since 2001 are the much-publicized deaths of two living liver donors and the introduction of the MELD allocation system around that same time $(7,8)$.

Age: Since 1996, when 95\% of the living donor transplants were performed on patients younger than 18 years old, the age distribution of LDLT recipients has shifted continuously toward older recipients. In 2005 , only $18 \%$ of LDLT recipients were pediatric candidates, $51 \%$ were 50 or older, and $8 \%$ were 65 or older (Figure 15).

Gender, race, ethnicity, blood type: The majority of LDLT recipients continue to be white. The percentage has risen slowly from $73 \%$ in 1995 to $77 \%$ in 2005 . There has been a steady decrease in the percentage LDLT recipients who are African American (4\% in 2005, down from 13\% in 1996 and $18 \%$ in 1998). The percentage of LDLT recipients who are Hispanic/Latino has dropped too, though not as much $(10 \%$ in 2005 , down from $13 \%$ in 1996 and $19 \%$ in 1999). Since 2000, most LDLT recipients have been male (58\% in 2005). The distribution of blood types is similar to that seen among recipients of DDLT and among the general population.

Diagnosis: The primary diagnosis of LDLT recipients has undergone considerable change. In 1996, most of cases were performed for biliary atresia $(61 \%)$, followed by acute hepatic necrosis $(18 \%)$, with noncholestatic liver disease representing $6 \%$ and malignancies $5 \%$. In 2005, only $7 \%$ were performed for biliary atresia. This shift most likely represents the implementation of the MELD/PELD system, which has increased the pediatric population's access 

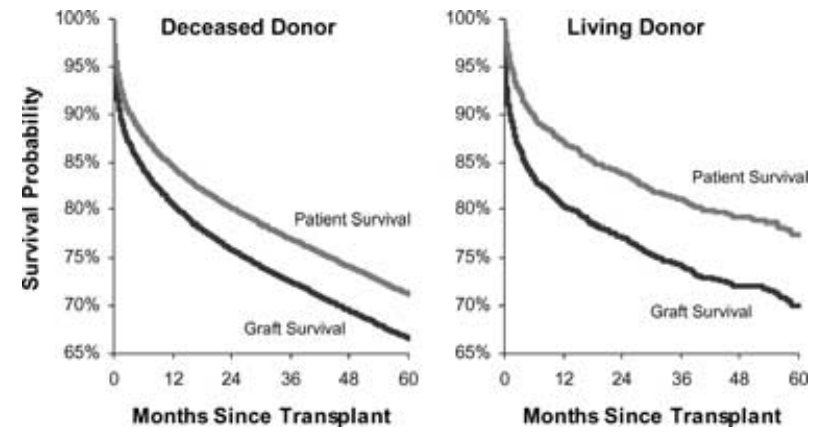

Includes all liver transplants from 1/1/96 through 12/31/05. Source: SRTR Analysis, November 2006.

Figure 16: Unadjusted patient and graft survival of liver recipients, by deceased and living donor.

to available deceased donor livers. In contrast, LDLT for noncholestatic disease increased to $46 \%$ in 2005 , a trend similar to that seen in recipients of deceased donor livers. Of note, $21 \%$ of LDLT recipients were transplanted for cholestatic disease in 2005 , compared to only $7 \%$ for DDLT recipients.

\section{Liver transplant patient survival}

Among the most recent transplant cohorts for whom follow-up data are available, patient survival following DDLT was $93 \%$ at 3 months, $87 \%$ at 1 year, $79 \%$ at 3 years and $73 \%$ at 5 years. These survival rates are adjusted for recipient age, gender, race and diagnosis at the time of transplant. The corresponding patient survival was slightly better for recipients of LDLT (96\%, 92\%, 83\% and 77\%, respectively). A very similar pattern can be noted when analyzing graft survival.

Figure 16 shows unadjusted patient and graft survival for all LDLT and DDLT recipients between 1996 and 2005. Al-

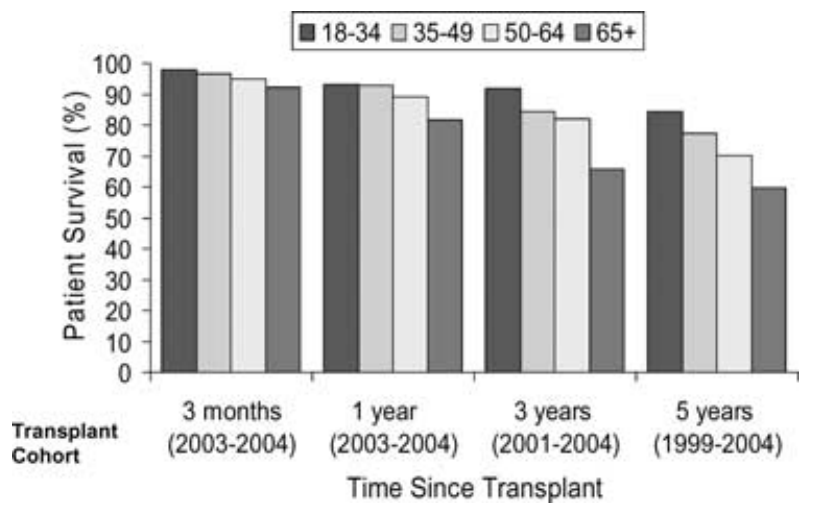

Source: 2006 OPTN/SRTR Annual Report, Table 9.12b.

Figure 17: Adjusted patient survival of living donor liver recipients at various time intervals, by age group.

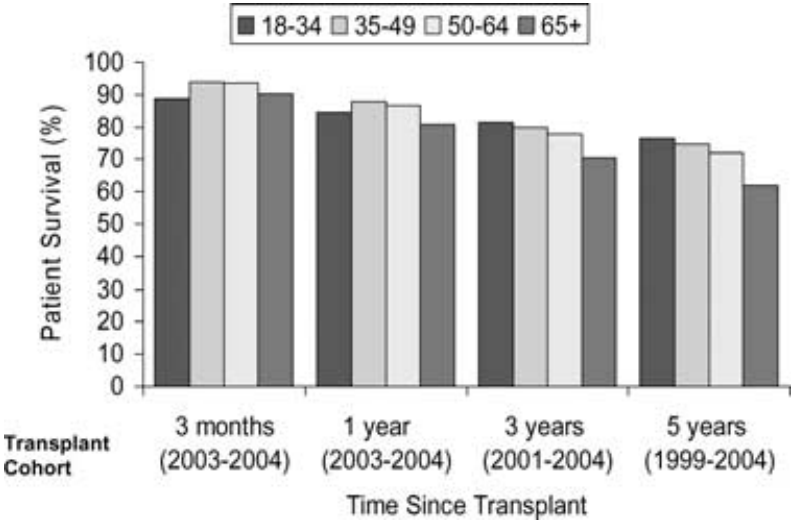

Source: 2006 OPTN/SRTR Annual Report, Table 9.12a.

Figure 18: Adjusted patient survival of deceased donor liver recipients at various time intervals, by age group.

though these statistics may suggest that results for LDLT are superior to those for DDLT, it is important to keep in mind that, in general, LDLT recipients have a lower acuity of illness (e.g. lower MELD score), and would thus be expected, other things being equal, to have better posttransplant outcomes. In addition, these 10-year cohorts subsume most or all of the learning curve effect that has been previously described for centers performing LDLT (9), so new centers embarking on LDLT programs might not necessarily have as good outcomes as shown here.

Demographic factors: Adjusted patient survival tended to decline with recipient age in adults who received LDLT, with the gap being more evident as the posttransplant time increases (Figure 17). The same pattern holds for long-term survival of DDLT recipients, while no age-related pattern is apparent in short-term survival (Figure 18). After 1 year, African Americans displayed lower survival rates

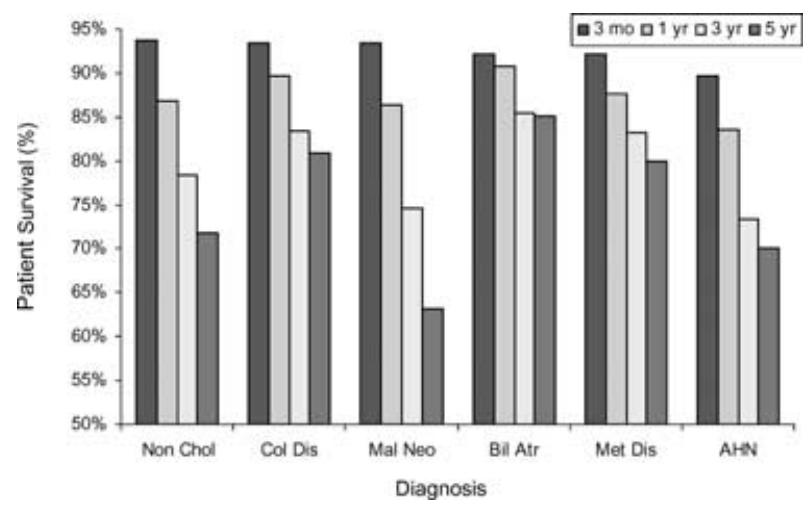

Source: 2006 OPTN/SRTR Annual Report, Table 9.12a

Figure 19: Adjusted patient survival of deceased donor liver recipients at various time intervals, by diagnosis. 


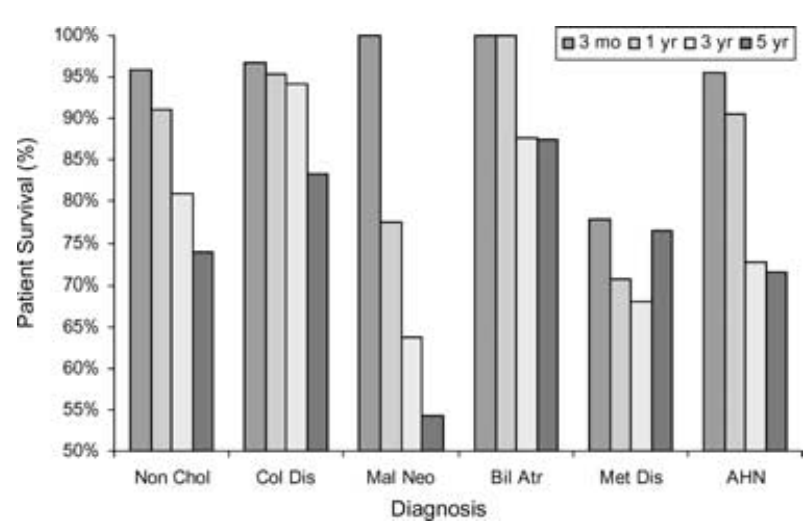

Source: 2006 OPTN/SRTR Annual Report, Table 9.12b

Figure 20: Adjusted patient survival of living donor liver recipients at various time intervals, by diagnosis.

(84\% at 1 year, $74 \%$ at 3 years, $65 \%$ at 5 years for DDLT recipients) than other racial groups (e.g. $87 \%, 79 \%$ and $74 \%$, respectively, for whites).

Medical factors: Adjusted patient survival at 3 months among DDLT recipients was similar regardless of the etiology of liver disease (between 90\% and 94\%). Patients whose main indication for transplant was biliary atresia exhibited the highest survival rates beyond 3 months $(91 \%$ at 1 year, $86 \%$ at 3 years, $85 \%$ at 5 years), followed by recipients with cholestatic liver disease and those with metabolic disease. Diagnoses of malignant neoplasm or acute hepatic necrosis were associated with reduced longterm survival probability $(75 \%$ at 3 years and $64 \%$ at 5 years for malignant neoplasm, respectively; $74 \%$ and $70 \%$ for acute hepatic necrosis), compared to other diagnoses (Figure 19). Survival rates for LDLT recipients showed some similar patterns (e.g. relatively high for biliary atresia and low for malignant neoplasm, 5 years after transplant), but were considerably lower for recipi-

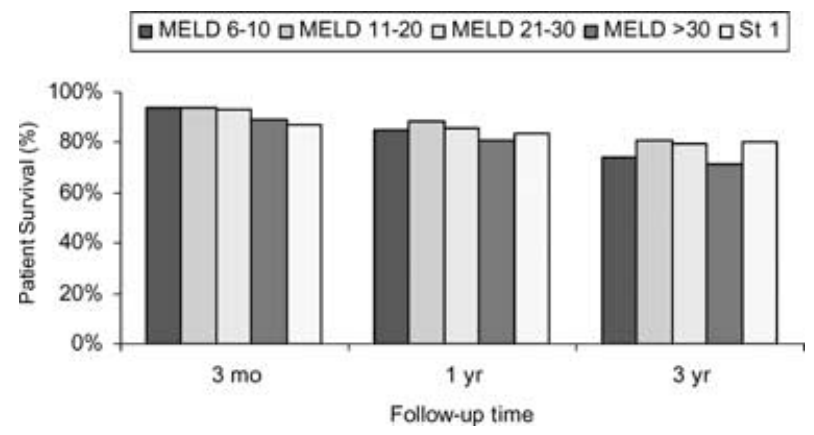

Source: 2006 OPTN/SRTR Annual Report, Table 9.14a

Figure 21: Unadjusted patient survival of deceased donor liver recipients at various time intervals, by severity of disease.

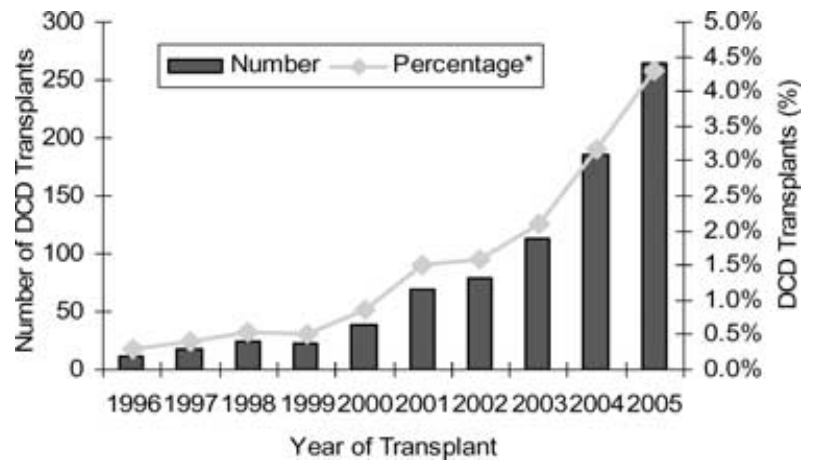

*As percentage of all DDLT.

Source: SRTR Analysis, May 2006.

Figure 22: Number and percentage of DCD liver transplants, by year, 1996-2005.

ents with metabolic disorder at every follow-up time point (Figure 20).

Unadjusted patient survival at 3 months was lower for patients who received a DDLT at Status $1(87 \%)$ or at MELD $>30(89 \%)$ than for patients who received a transplant at low or intermediate MELD scores (Figure 21). Further out from the time of transplant, the discrepancy between Status 1 recipients and MELD 11-20 recipients decreased (84\% vs. $89 \%$, respectively, at 1 year; $80 \%$ vs. $81 \%$ at 3 years) and recipients who were at MELD >30 at time of transplant fared worse ( $72 \%$ at 3 years). It is interesting to note that DDLT recipients with MELD scores 6-10 at transplant had good short-term survival, while their survival at 3 years was only slightly better than that of recipients with high MELD (76\% at 3 years).

\section{Donation After Cardiac Death Liver Transplants}

There were 826 DCD liver transplants among the 48593 DDLT performed between 1996 and 2005. While the overall proportion of DCD liver transplants was $1.7 \%$ of all DDLT over the decade, the annual number of DCD liver transplants increased from $0.3 \%$ in $1996(n=12)$ to $4.3 \%$ in 2005 ( $n=264$ ) (Figure 22). The proportion of DCD donors from whom a liver was recovered increased from $38 \%$ in 1996 to $70 \%$ in 2005, suggesting dissemination of this approach to liver donation throughout the transplant community. The number of transplant programs performing DCD liver transplants increased from 7 in 1996 to 33 in 2005 (Table 2).

Graft and patient outcomes following DCD liver transplantation have been reported to be inferior to those using donation after brain death (DBD) donors (8,10-12). However, there is some preliminary evidence that changes in practice are leading to improved results for DCD liver trans- 
Table 2: Number of centers that performed at least one DCD liver transplant, by year (1996-2005)

\begin{tabular}{lc}
\hline Year & Number of centers \\
\hline 1996 & 7 \\
1997 & 10 \\
1998 & 9 \\
1999 & 7 \\
2000 & 8 \\
2001 & 17 \\
2002 & 21 \\
2003 & 29 \\
2004 & 31 \\
2005 & 33 \\
\hline
\end{tabular}

Source: SRTR Analysis, May 2006.

plants. In 2003, 1-year patient survival rates after DCD and DBD liver transplants were $76 \%$ and $85 \%$, respectively, but they were nearly identical (86.1\% vs. 85.9\%) in 2004 (Figure 23). Some of the improvement in patient survival might be attributable to more prompt or effective retransplantation after DCD liver graft failure, but graft survival data appear to parallel the patient survival results (Figure 24).

While these early trends cannot be rigorously assessed, it suggests that the adverse effect of a DCD liver transplant on early outcome may be abating. At a recent consensus conference on donation after cardiac death (DCD), it was averred that limiting warm ischemia time in the donor between withdrawal of support and declaration of death to 30 min (vs. the 60-min limit generally used for DCD kidney recovery) might have a beneficial effect on DCD liver function (13). Mean warm ischemia time has decreased slightly (from 19 to $16 \mathrm{~min}$ ) over the past few years; more important, the proportion of DCD livers recovered after more than 30 min has dropped from $16 \%$ to $10 \%$.

The effect of warm ischemia time on outcome is clearly seen in a multivariable Cox regression model of time to graft failure after DCD liver transplant. After adjustments for recipient factors and year of transplant, each 5-min pe-

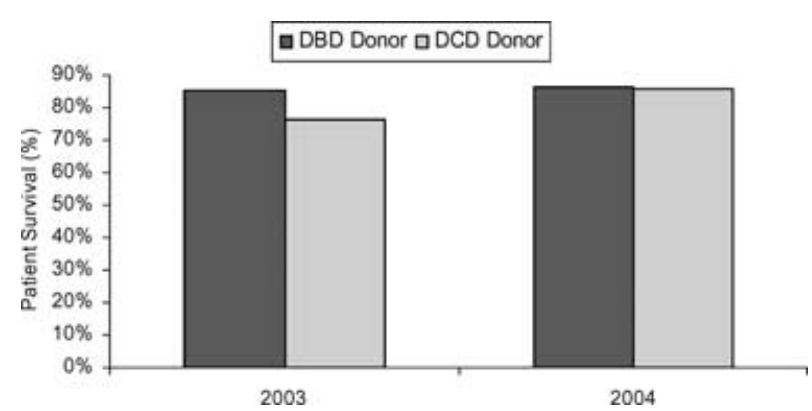

Source: SRTR Analysis, May 2006.

Figure 23: Patient survival of DCD donors and DBD donors at 1 year following liver transplantation, 2003-2004.

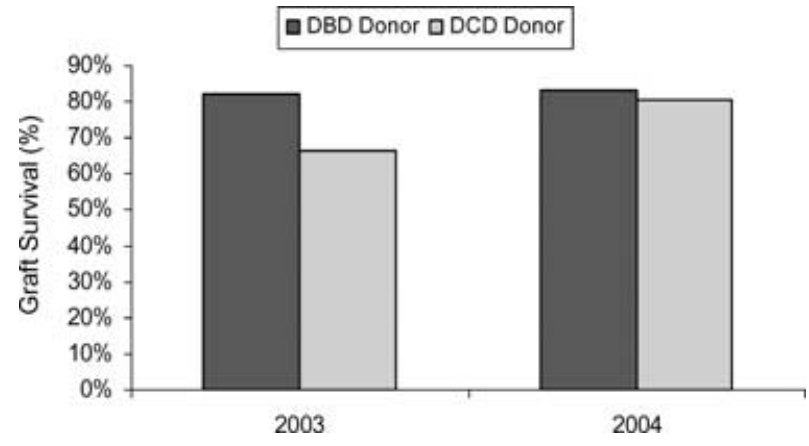

Source: SRTR Analysis, May 2006.

Figure 24: Graft survival of DCD donors and DBD donors at 1 year following liver transplantation, 2003-2004.

riod of warm ischemia time was associated with a $16 \%$ higher risk of graft failure (hazard ratio 1.157; 95\% confidence interval 1.070-1.250; $p=0.0002$ ).

It has been suggested that long-term outcomes after DCD liver transplantation will be adversely affected by the development of ischemic-type biliary strictures (10). Continued observation of recipients of DCD liver transplants is thus warranted to determine whether recent improvements in short-term outcome will be sustained over time.

\section{Simultaneous Liver-Kidney Transplantation}

An increase in simultaneous liver-kidney (SLK) transplantation coincided with the introduction of MELD prioritization for liver transplant. Hepatorenal syndrome has become an increasingly important indication for liver transplantation, and liver transplant candidates with intrinsic renal disease are receiving transplants at increased rates. In fact, a liver transplant candidate on dialysis starts with a MELD

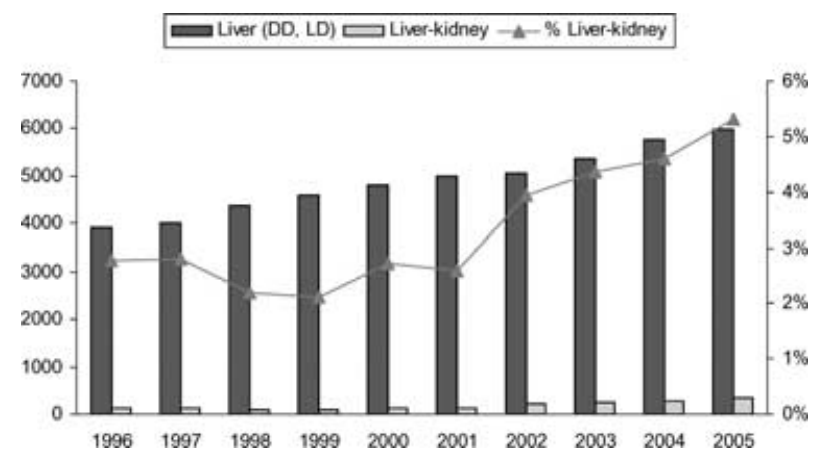

Source: Draft 2006 OPTN/SRTR Annual Report, Tables 1.7 and 1.8.

Figure 25: Recipients of liver and SLK transplants by year, 1996-2005. 


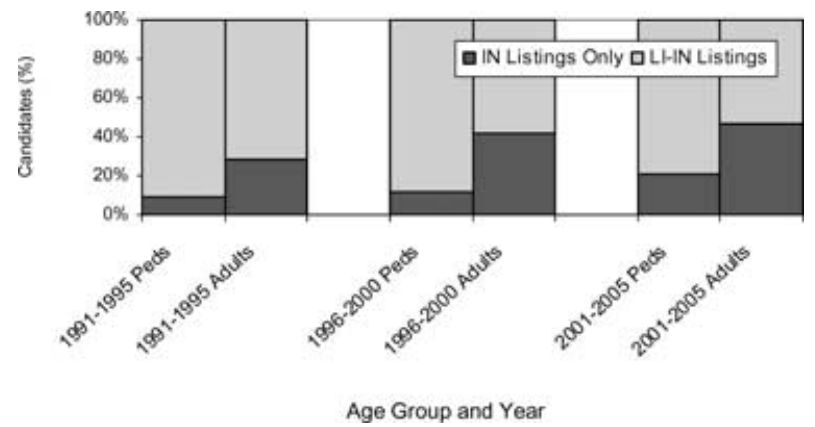

Source: SRTR Analysis, May 2006.

Figure 26: Distribution of candidates listed for intestine versus liver-intestine transplant, by age group and year, 19912005.

score of 20 even before INR and serum bilirubin levels are added to the MELD score calculation. As serum creatinine is heavily weighted in the equation used to calculate MELD scores, renal dysfunction has become increasingly present in liver transplant recipients.

In the first year of the MELD allocation system, the number of SLK transplants rose by more than 50\% (134 in 2001, 210 in 2002). As shown in Figure 25, since the introduction of MELD allocation, the percentage of liver transplants performed as SLK transplants has continued to increase $15.3 \%$ in 2005 vs. $2.6 \%$ in 2001 ). Nearly $10 \%$ of liver recipients were on dialysis at the time of transplant, received an SLK transplant, or both (SRTR analysis; data not shown).

It is important to note that in the OPTN allocation rules, listing for a solitary kidney transplant requires a calculated glomerular filtration rate (GFR) of less than $20 \mathrm{~mL} / \mathrm{min}$, yet there is no GFR listing threshold for SLK (14). This difference in criteria has led to a difference in the degree of renal impairment at transplant between SLK and solitary kidney recipients. In fact, only about $60 \%$ of those receiving SLK are on dialysis at transplantation (SRTR analysis;

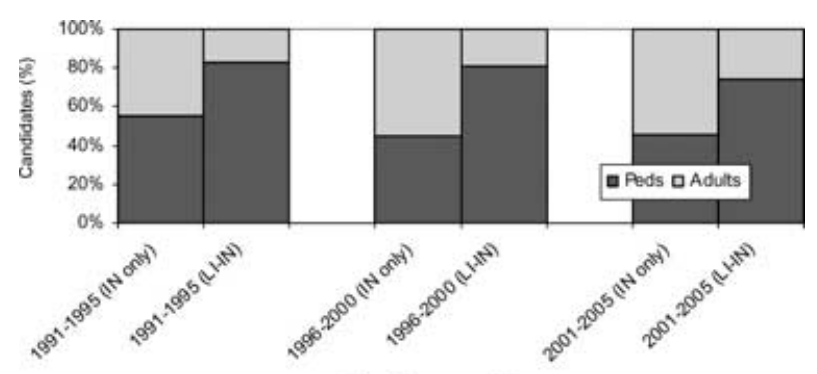

Age Group and Era

Source: SRTR Analysis, May 2006.

Figure 27: Age distribution of candidates listed for intestine versus liver-intestine transplant, by year, 1991-2005.

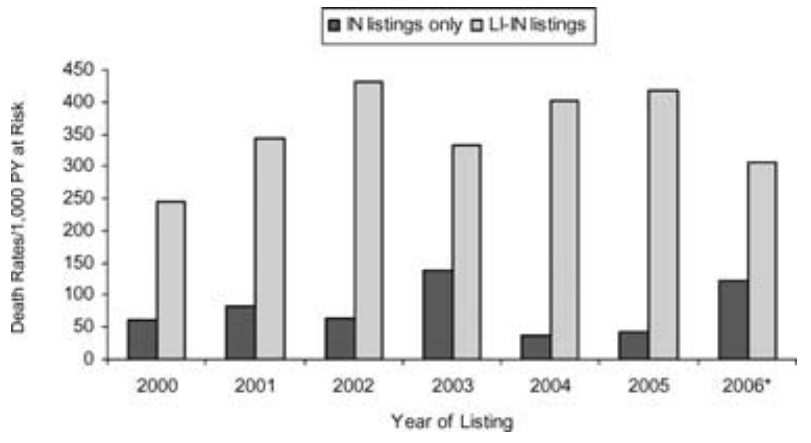

* Death rates for 2006 are based on a very low reported number of deaths and therefore are highly unstable Source: SRTR Analysis, May 2006.

Figure 28: Death rates of candidates listed for an intestine or liver-intestine transplant, by year, 2000-2006.

data not shown). These facts are disturbing to those primarily involved with kidney transplantation. With the heavy weighting of serum creatinine in the MELD score, and the subsequent increased priority of those with significant renal dysfunction and increased number of SLK transplants, a call has been made for consistent evaluation and selection criteria for SLK transplants in those liver transplant candidates with renal insufficiency.

\section{Intestine Transplantation}

Intestines are the least transplanted abdominal organ, but the rate of intestine transplantation is increasing the most. Although significant problems persist, intestine transplant outcomes have steadily improved, as detailed below. Because of the small number of such transplants, we have grouped together sequential 5-year eras of transplants when discussing trends: 1991-1995, 1996-2000 and 2001-2005.

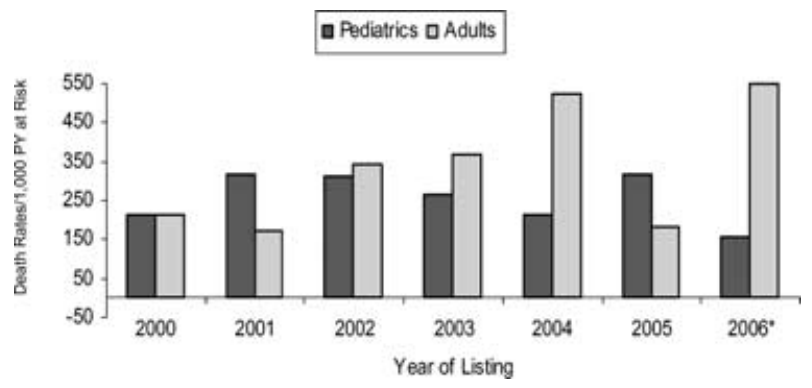

*Death rates for 2006 are based on a very low reported number of deaths and therefore are highly unstable Source: 2006 OPTN/SRTR Annual Report, Table 10.3.

Figure 29: Death rates on the waiting list for candidates listed for an intestine transplant, by age group and year, 2000-2006. 
Table 3: Death rates for candidates listed for an intestine transplant, by liver listing and age group

\begin{tabular}{lcc}
\hline $\begin{array}{l}\text { Age group and } \\
\text { listing combination }\end{array}$ & $\begin{array}{c}\text { Number of } \\
\text { deaths }\end{array}$ & $\begin{array}{c}\text { Annual death rates per } \\
1000 \text { patient-years at risk }\end{array}$ \\
\hline Pediatrics-intestine only & 13 & 62.2 \\
Pediatrics-liver/intestine & 201 & 351.5 \\
Adults-intestine only & 18 & 153.2 \\
Adults-liver/intestine & 56 & 475.8 \\
\hline
\end{tabular}

Source: SRTR Analysis, May 2006.

\section{Intestine waiting list}

Intestine transplant listings have increased significantly. The number of listings has increased from 236 (1991$1995)$ to 1161 (2001-2005). Since previous results have indicated that most intestinal transplants have been performed together with a liver transplant, we have separated all candidates listed for intestinal transplants into two subsets: all candidates listed for both a liver and an intestine, and candidates listed only for an intestine and never for a liver. In the three sequential eras, liver-intestine candidates have made up $87 \%, 80 \%$ and $70 \%$ of the waiting list population, respectively, indicating that while most intestine listings were combined with a liver listing in all eras, this practice has been decreasing (Figure 26). Alternatively, when the intestinal waiting list population was divided by age, pediatric candidates made up $79 \%, 74 \%$ and $66 \%$, respectively (Figure 27). While pediatric patients are listed for intestine transplants more often than adults, this gap is progressively narrowing.

Waiting list mortality has been consistently higher with candidates waiting for intestine transplants than for candidates waiting for any other organ transplant, primarily reflecting the outcomes of the liver-intestine candidate subset (Figure 28). Although there has been significant annual variability, in general waiting list mortality appears to be stable among pediatric candidates and increasing in adults (Figure 29). When waiting list mortality rates since 2000 were compared, there was a 5.7-fold higher mortality among pediatric liver-intestine candidates compared to the intestine-

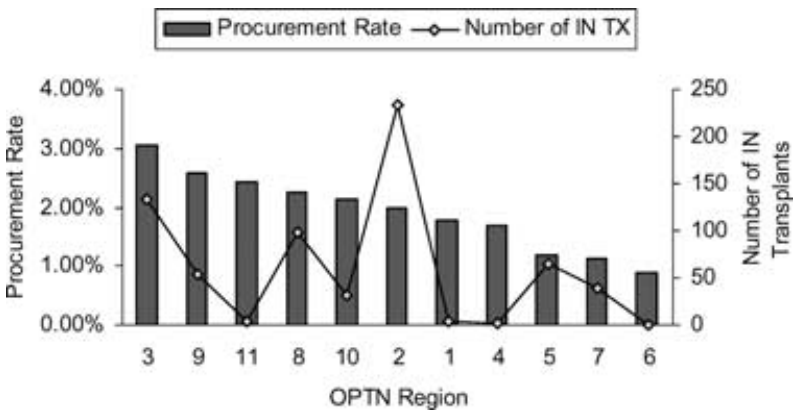

Source: SRTR Analysis, May 2006.

Figure 30: Percentage of intestines procured and transplanted, by OPTN region, 2001-2005.

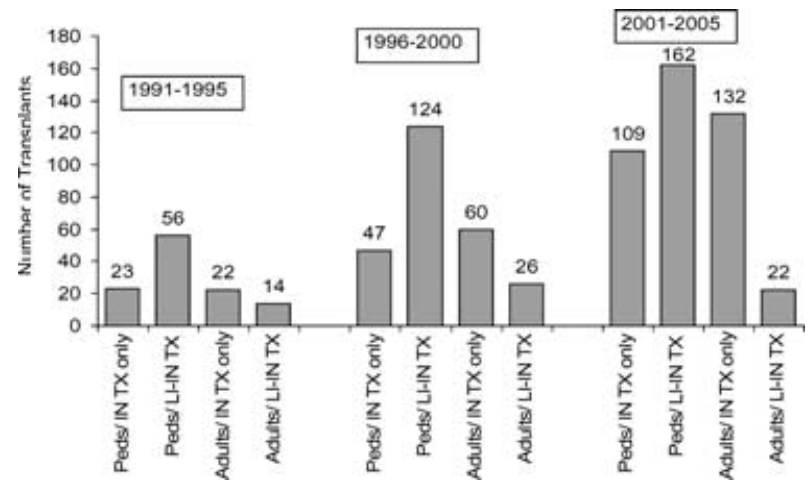

Source: SRTR Analysis, May 2006.

Figure 31: Number of intestine and liver-intestine transplants performed, by age group and year, 1991-2005.

only candidates. Adult liver-intestine-candidates had a 3.1fold higher mortality than adult intestine-only candidates (Table 3).

Intestine waiting list mortality was higher for adults than for pediatric candidates. Among intestine-only candidates, adults had a 2.5-fold higher mortality rate than pediatric candidates. Similarly, among liver-intestine candidates, adults had a mortality rate 1.4-fold higher than that of pediatric candidates.

\section{Intestine procurement}

Intestine procurements have increased since 2000 although the percentage of multi-organ donors from whom an intestine was procured remains low (2\%). To put this into perspective, $0.22 \%$ of patients on the waiting list at the end of 2005 were waiting for an intestine graft, while $0.64 \%$ of transplants performed in 2005 involved an intestine transplant. There is significant variability between regions regarding the percentage of donors from whom an

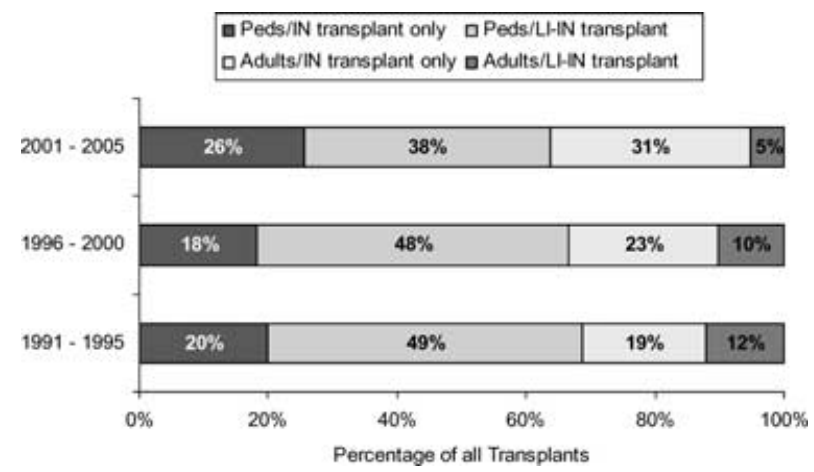

Source: SRTR Analysis, May 2006.

Figure 32: Distribution of intestine and liver-intestine transplants, by age group and year, 1991-2005. 


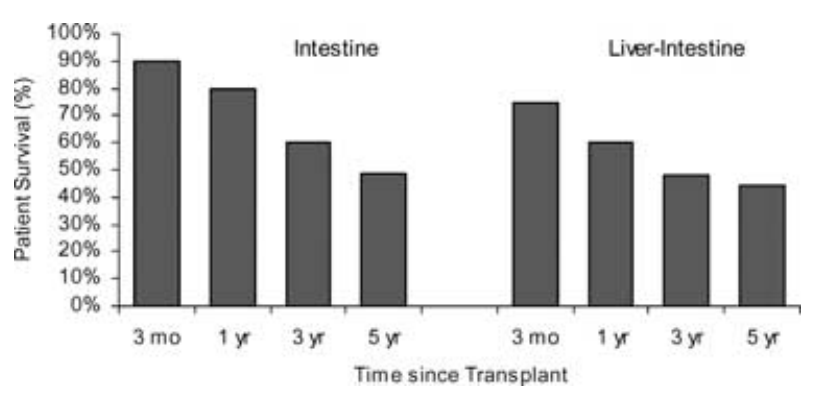

Source: SRTR Analysis, May 2006.

Figure 33: Adjusted patient survival following intestine transplantation, 1991-2005.

intestine was procured, from 3.0\% (Region 3) to 0.9\% (Region 9) in 2001-2005 (Figure 30). Over the same period, the most intestinal transplants (234) were performed in Region 2 and the fewest (none) were performed in Region 6.

\section{Intestine transplantation}

The number of intestine transplants performed has been steadily increasing: total transplant numbers have increased from 115 (1991-1995) to 425 (2001-2005) (Figure 31). While most intestine transplants performed since 1990 have included a liver (51\%), this trend has been decreasing, with combined liver-intestine transplants representing $61 \%, 58 \%$ and $43 \%$ of intestine transplants over the three periods examined. Intestinal transplants continue to be more commonly performed in pediatric patients (64\% in 2001-2005, down from $69 \%$ in 1991-1995). In the most recent era (2001-2005), pediatric intestine transplants combined with a liver were the most common intestine transplant performed overall (38\%): $60 \%$ of all pediatric intestine transplants were combined liver-intestine transplants. Adult intestine-only transplants are the second most common intestine transplant performed overall (31\%): $86 \%$ of all adult intestine transplants were intestineonly (Figure 32).

\section{Intestine graft and patient survival}

Following transplantation, there were outcome differences between patients who received only an intestine and those

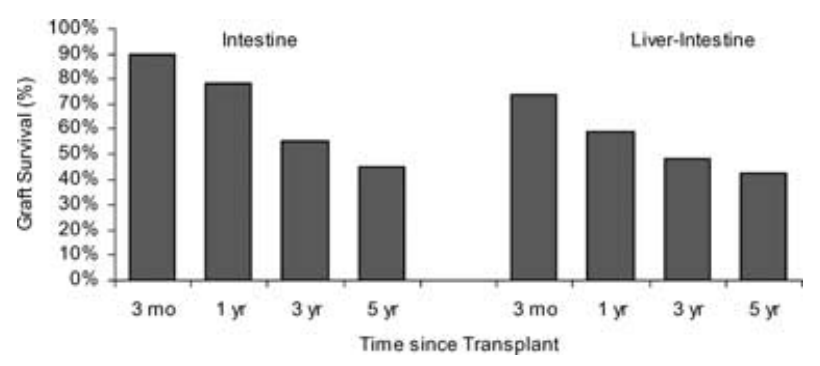

Source: SRTR Analysis, May 2006.

Figure 34: Adjusted graft survival following intestine transplantation, 1991-2005. who received both an intestine and a liver. When adjusted for age, race and diagnosis, post-transplant graft and patient survival data were superior for intestine-only recipients; these differences were most apparent at 1 year following transplantation but diminished at subsequent time intervals (Figures 33 and 34). The early survival disparity likely reflects the fact that the intestine-liver recipients were much sicker at the time of transplant, as demonstrated by their higher waiting list mortality, and the fact that they undergo more extensive surgery than the intestine-only recipients. The subsequent narrowing of the gap in graft and patient survival between the two groups may reflect reduced graft loss due to chronic rejection in the intestine-liver recipient population, although conclusive evidence for the immunoprotective effect of the liver on the intestine graft has been difficult to demonstrate.

\section{Conclusion}

Transplantation continues to be a very successful treatment for end-stage liver disease. The number of liver transplants has increased steadily over the last decade, with 6441 procedures performed in 2005. This growth may reflect a larger number of deceased donors, especially in the 'expanded donor criteria' and DCD categories. Although the long-term outcomes for these recipients remain unclear, short-term benefits have been clearly identified. Patient and graft survival for recipients of DCD grafts have continued to improve, with 1-year survival rates for recipients of DCD grafts in 2004 virtually identical to those seen for recipients of standard criteria grafts. Warm ischemia time has been identified as a negative prognostic indicator, and procurement of livers more than 30 min after withdrawal of life support now occurs less than $10 \%$ of the time.

The MELD/PELD allocation system continues to be refined. The 'Share 15' policy, which mandates sharing of available organs within a region so that patients with MELD scores $\geq 15$ receive priority, was implemented in January of 2005. Subsequently, the number of DSAs transplanting $90 \%$ of their recipients at MELD scores $\geq 15$ increased substantially from $27(55 \%)$ to 42 (86\%). However, there was no significant increase in sharing between DSAs, suggesting that available organs were being used for candidates with higher MELD scores within the DSA of origin.

LDLT has emerged as a viable alternative to deceased donor transplantation in the past decade, yet there is a great deal of regional variation in how much it is employed. A trend toward use in regions with low availability of deceased donors and high average MELD score at transplant may in part explain the regional variability. In the past decade, LDLT has changed from predominantly parent-tochild to adult-to-adult LDLT. Patient and graft survival rates for recipients of LDLT are now equivalent to and in some cases slightly better than those seen for recipients of standard criteria grafts. 
Activity in all areas of intestine transplantation continues to increase. Data now available for recipients of intestinealone transplants suggest that patient and graft survival of this group is superior to that seen for recipients of liverintestine grafts in the first posttransplant year. An immunoprotective effect of the liver may be noted in combined recipients in subsequent years.

\section{Acknowledgment}

The Scientific Registry of Transplant Recipients is funded by contract number 234-2005-37009C from the Health Resources and Services Administration (HRSA), U.S. Department of Health and Human Services. The views expressed herein are those of the authors and not necessarily those of the U.S. Government. This is a United States Government-sponsored work. There are no restrictions on its use.

This study was approved by HRSA's SRTR project officer. HRSA has determined that this study satisfies the criteria for the IRB exemption described in the 'Public Benefit and Service Program' provisions of 45 CFR 46.101(b)(5) and HRSA Circular 03.

Note on sources: The articles in this report are based on the reference table in the 2006 OPTN/SRTR Annual Report, which are not included in this publication. Many relevant data appear in the figures and tables included here; other table from the Annual Report that serve as the basis for this article include the following: Tables 1.6, 9, 1a-b, 9.2a, 9.3, 9.4a-b, 9.7a, 9.10a-b, 9.12a-b, 9.14a-b, and 10.3. All of these tables may be found online at: http://www.ustransplant.org.

\section{References}

1. Roberts JP, Dykstra DM, Goodrich NP et al. Geographic differences in event rates by model for end-stage liver disease score. Am J Transplant 2006; 6: 2470-2475.
2. Meier-Kriesche H-U, Li S, Gruessner RWG et al. The 2005 SRTR report on the state of transplantation: immunosuppression: evolution in practice and trends, 1994-2004. Am J Transplant 2006; 6: 1111-1131.

3. Magee JC, Barr ML, Basadonna GP et al. Repeat transplantation, 1996-2005. Am J Transplant 2007; 7: 1424-1433.

4. Merion RM, Schaubel DE, Dykstra DM et al. The survival benefit of liver transplantation. Am J Transplant 2005; 5: 307-313.

5. Organ Procurement and Transplantation Network. Policy 3.6. Allocation of livers. http://www.optn.org/PoliciesandBylaws/ policies/pdfs/policy 8.pdf (Accessed August 3, 2006).

6. Merion RM, Goodrich NP, Freeman RB et al. Impact of a regional allocation rule for high MELD/PELD liver transplant candidates. Liver Transpl 2006; 12: C103.

7. Brown RS Jr, Russo MW, Lai M et al. A survey of liver transplantation from living adult donors in the United States. N Engl J Med 2003; 348: 818-825.

8. Malagó M, Testa G, Marcos A et al. Ethical considerations and rationale of adult-to-adult living donor liver transplantation. Liver Transpl 2001; 7: 921-927.

9. Olthoff KM, Merion RM, Ghobrial RM et al. Outcomes of 385 adult-to-adult living donor liver transplant recipients: a report from the A2ALL Consortium. Ann Surg 2005; 242: 314-323.

10. D'Alessandro AM, Fernandez LA, Chin LT et al. Donation after cardiac death: The University of Wisconsin experience. Ann Transplant 2004; 9: 68-71

11. Feng S, Goodrich NP, Bragg-Gresham JL et al. Characteristics associated with liver graft failure: the concept of a donor risk index. Am J Transplant 2006; 6: 783-790.

12. Merion RM, Pelletier SJ, Goodrich NP, Englesbe MJ, Delmonico FL. Donation after cardiac death as a strategy to increase deceased donor liver availability. Ann Surg 2006; 24: 555562.

13. Bernat JL, D'Alessandro AM, Port FK et al. Report of a national conference on donation after cardiac death. Am J Transplant 2006: 6: $281-291$

14. Organ Procurement and Transplantation Network. Policy 3.5. Allocation of deceased kidneys. http://www.optn.org/ PoliciesandBylaws2/policies/pdfs/policy 7.pdf (Accessed November 30, 2006). 